\title{
LncRNA myocardial infarction-associated transcript promotes cell proliferation and inhibits cell apoptosis by targeting miR-330-5p in epithelial ovarian cancer cells
}

\author{
Shiqing Shao, Jun Tian, Hongxia Zhang, Shelian Wang
}

Department of Obstetrics and Gynecology, Huaihe Hospital of Henan University, Kaifeng, Henan Province, China

Submitted: 16 January 2018

Accepted: 22 March 2018

Arch Med Sci 2018; 14, 6: 1263-1270

DOI: https://doi.org/10.5114/aoms.2018.75535

Copyright $\odot 2018$ Termedia \& Banach

\section{Abstract}

Introduction: Long non-coding RNAs (IncRNAs) have been shown to have great importance in cancer development and progression. However, the mechanism of IncRNAs in epithelial ovarian cancer remains unclear. In the present study, we aimed to explore the role of the IncRNA myocardial infarction-associated transcript (MIAT) in epithelial ovarian cancer tumorigenesis. Material and methods: Quantitative real-time PCR (qRT-PCR) was used to determine MIAT expression in human epithelial ovarian cancer tissues and cell lines, and the effects of MIAT on cell proliferation and cell apoptosis were determined by CCK- 8 assay or flow cytometry analysis. Dual-Luciferase Reporter assay and Western blot assay were used to explore the molecular mechanisms of MIAT in epithelial ovarian cancer cells progression.

Results: Our data showed that the expression of IncRNA MIAT was remarkably increased in human epithelial ovarian cancer tissues and cell lines $(p<0.05)$. High MIAT expression was associated with poor overall survival of epithelial ovarian cancer patients $(p<0.05)$. Function assays showed that knockdown of MIAT expression significantly inhibited epithelial ovarian cancer cell proliferation and promoted cell apoptosis in vitro $(p<0.05)$. Moreover, we revealed that MIAT might function as an endogenous miR-330-5p sponge to regulate the target gene of miR-330-5p in epithelial ovarian cancer progression.

Conclusions: LncRNA MIAT was found to be a tumor oncogenic IncRNA in epithelial ovarian cancer tumorigenesis. LncRNA MIAT promoted cell proliferation and inhibited cell apoptosis by negative regulation of miR-330-5p in epithelial ovarian cancer cells. Our findings suggested that MIAT might act as a candidate prognostic biomarker and new therapeutic target for treating epithelial ovarian cancer patients.

Key words: long non-coding RNAs, MIAT, miR-330-5p, epithelial ovarian cancer.

\section{Introduction}

Epithelial ovarian cancer (EOC), one of the most deadly gynecological malignancies, affected approximately 239000 people and caused an estimated 151900 deaths during 2012 according to 2015 global cancer statistics, accounting for about $5 \%$ of all cancer and $4.2 \%$ of all cancer deaths among females worldwide [1, 2]. Despite the great improvement of early diagnosis and optimal treatment, overall survival of EOC patients

\author{
Corresponding author: \\ Shiqing Shao \\ Department of Obstetrics \\ and Gynecology \\ Huaihe Hospital \\ of Henan University \\ 8 Baogonghu North Road \\ Kaifeng Henan Province \\ 475000, China \\ E-mail: shiqings0625@163.com
}


remains unsatisfactory [3]. Therefore, demonstrating the molecular mechanism underlying EOC progression is necessary for development of successful therapeutic strategies to improve EOC patients' survival.

Long non-coding RNAs (IncRNAs) are a new class of non-coding RNAs ( $>200$ nucleotides) with limited or no protein-coding capacity [4]. With the continuous advances of research approaches, dysregulation of IncRNAs was found to contribute to various malignant tumors, including EOC progression. For example, Zhu et al. found that downregulation of InCRNA TUBA4B was associated with poor prognosis for EOC [5]. Jin et al. showed that InCRNA MALAT1 promoted proliferation and metastasis in EOC via the PI3K-AKT pathway [6]. Zhang et al. suggested that IncRNA HOXD-AS1 promoted epithelial ovarian cancer cell proliferation and invasion by targeting miR-133a-3p and activating the $W n t / \beta$-catenin signaling pathway [7]. However, the roles of IncRNAs in EOC progression are still largely unclear.

Myocardial infarction-associated transcript (MIAT) is one of the noncoding RNAs first identified as an IncRNA in 2006 [8]. Recent studies showed that MIAT plays important roles in microvascular dysfunction [9], myocardial infarction [10], and diabetic retinopathy [11]. Moreover, accumulating evidence has proven that MIAT plays an oncogenic role in tumor progression. However, the roles and underlying mechanism of MIAT in EOC are still unclear.

In the present study, we explored the role of InCRNA MIAT in promoting EOC cell proliferation and apoptosis. In addition, we investigated whether MIAT affected the biological processes of EOC via regulating the miRNA expression. Taken together, our findings suggested that MIAT promoted EOC cell progression through inhibiting miR-330-5p expression.

\section{Material and methods}

\section{Patients and tissue samples}

We collected 53 samples of surgical EOC tissues and 19 samples of normal ovarian tissues at the Department of Gynecology of Huaihe Hospital of Henan University between 2011 and 2012. The tissue samples were confirmed by pathological examination and immediately stored in liquid nitrogen after surgery. Written informed consent was obtained from individual patients prior to surgery. The study was approved by the Ethics Committee of Huaihe Hospital of Henan University.

\section{Cell culture and transfection}

Human EOC cell lines (SKOV3, OVCAR3, $\mathrm{HO} 8910$, and A2780) were purchased from Amer- ican Type Culture Collection (ATCC, Rockville, USA). The normal cell line human ovarian surface epithelial (HOSE) was purchased from the Cell Bank of the Chinese Academy of Science (Shanghai, China). All cells were cultured in RPMI-1640 (Invitrogen, USA) supplemented with $10 \%$ fetal bovine serum (FBS, Gibco, USA) and 100 units/ $\mathrm{ml}$ penicillin-streptomycin (Invitrogen, USA), and maintained at $37^{\circ} \mathrm{C}$ in a humidified incubator with $5 \% \mathrm{CO}_{2}$. siRNA against MIAT was designed and synthesized by Shanghai Genechem Co., Ltd. MiR-330-5p mimics and miR-330-5p inhibitors were obtained from Genepharma Co., Ltd. Cell transfection was performed with Lipofectamine 2000 (Invitrogen, USA) according to the manufacturer's instructions.

\section{RNA isolation and quantitative real-time PCR}

TRIzol Reagent (Invitrogen, USA) was used to extract total RNA according to the manufacturer's protocol. The quality and concentration of RNA were determined using a NanoDrop 2000 spectrophotometer (NanoDrop Technologies, USA). QRTPCR was performed using SYBR Premix ExTaq (TaKaRa, Dalian, China) with the ABI Prism 7900HT thermocycler (Applied Biosystems, USA). GAPDH was employed as the internal control for mRNA quantification. The primers used in the present study were as follows: MIAT forward primer 5'-TTTACTTTAACAGACCAGAA-3' and reverse primer 5'-CTCCTTTGTTGAATCCAT-3'; GAPDH forward primer 5'-CCACATCGCTCAGACACCAT-3' and reverse primer 5'-CCAGGCGCCCAATACG-3'. The relative expression was calculated using the $2^{-\Delta \Delta C T}$ method.

\section{Cell proliferation assay}

Cell proliferation was determined using a Cell Counting Kit-8 (CCK-8, Dojindo, Japan) assay. Twenty-four $\mathrm{h}$ later with transfection, cells were seeded into 96-well plates at a density of 5000 cells per well with $100 \mu \mathrm{l}$ of medium and continued to incubate at $37^{\circ} \mathrm{C}$. At $24 \mathrm{~h}, 48 \mathrm{~h}, 72 \mathrm{~h}$, and $96 \mathrm{~h}, 100 \mu \mathrm{l}$ of serum-free culture medium and $10 \mu \mathrm{l}$ of CCK-8 solutions were added to each well, followed by incubation at $37^{\circ} \mathrm{C}$ for $1 \mathrm{~h}$. The absorbance was measured with a plate reader at $450 \mathrm{~nm}$ on an enzyme-linked immunosorbent assay reader. Five independent samples were detected in each experimental group.

\section{Colony formation assay}

Cells $\left(1 \times 10^{3}\right)$ were seeded into each well of a 6-well plate in quadruplicate. Cells were cultured for 2 weeks in a $37^{\circ} \mathrm{C}$ incubator. Cells were washed with PBS and fixed with $4 \%$ paraformal- 
dehyde, stained with $1 \%$ crystal violet for $20 \mathrm{~min}$, and then washed with water. Then, the number of colonies was counted manually.

\section{Cell apoptosis assay}

The annexin V/PI assay was performed according to the manufacturer's instructions (Invitrogen, USA). Briefly, transfected cells were plated into 6-well plates for $24 \mathrm{~h}$. Afterwards, cells were washed with pre-cold PBS, trypsinized, and re-suspended in $100 \mu \mathrm{l}$ of binding buffer with $2.5 \mu \mathrm{l}$ of FITC conjugated annexin- $v$ and $1 \mu \mathrm{l}$ of PI $(100 \mu \mathrm{g} /$ $\mathrm{ml})$. Cells were then incubated at room temperature for $15 \mathrm{~min}$ in darkness. A total of at least 10000 cells were collected and calculated by flow cytometry (BD Biosciences, USA).

\section{Luciferase assay}

We cloned wild-type MIAT with potential miR330-5p binding sites or mutants of each site into pMIR-REPORT plasmids (Promega, USA). HEK293T cells were placed on a 24-well plate and co-transfected with luciferase plasmids and miR-330-5p mimics or control miRNA. After $48 \mathrm{~h}$ of transfection, firefly and Renilla luciferase activities were detected with the Dual-Luciferase Reporter Assay System (Promega, USA).

A

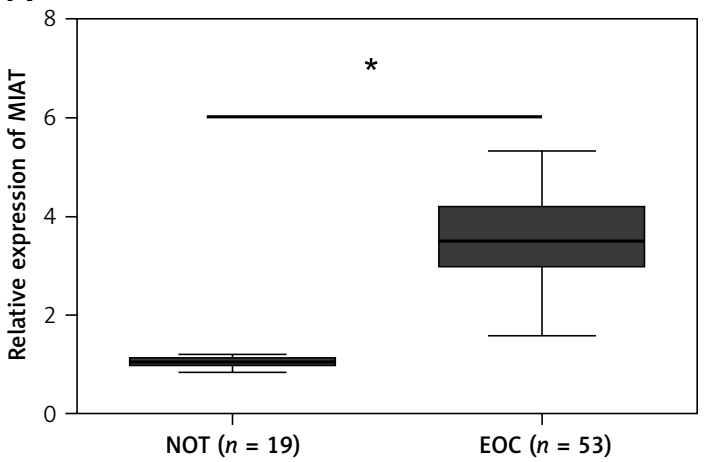

C

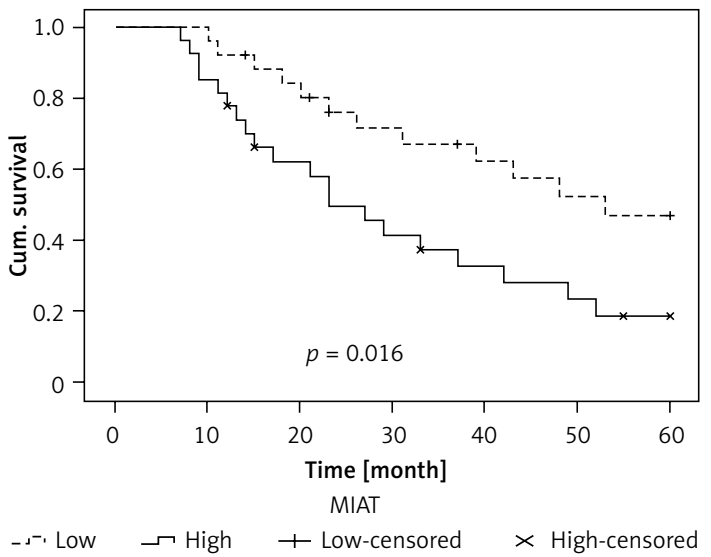

\section{Statistical analysis}

Statistical analysis were performed using SPSS 17.0. Data were presented as mean \pm standard deviation (SD). The Kaplan-Meier method was used to calculate the survival curve, and log-rank test to determine statistical significance. Student's $t$-test or $\chi^{2}$ test was used to evaluate the statistical significance of differences among different samples. $P<0.05$ was considered to indicate a statistically significant difference.

\section{Results}

\section{LncRNA MIAT was upregulated in EOC}

To explore the role of IncRNA MIAT in EOC progression, we firstly used qRT-PCR to determine MIAT expression in EOC tissues and cell lines. The results showed that MIAT expression was highly expressed in EOC tissues compared with normal ovarian tissues (NOT) (Figure 1 A; $p<0.05$ ). Next, we explored MIAT expression in EOC cell lines. QRT-PCR showed that MIAT expression was significantly higher in EOC cells (SKOV3, OVCAR3, HO8910, and A2780) than normal cell line human ovarian surface epithelial cells (HOSE) (Figure $1 \mathrm{~B}$; $p<0.05)$. In addition, according to the median relative MIAT expression value in EOC, we classi-

B

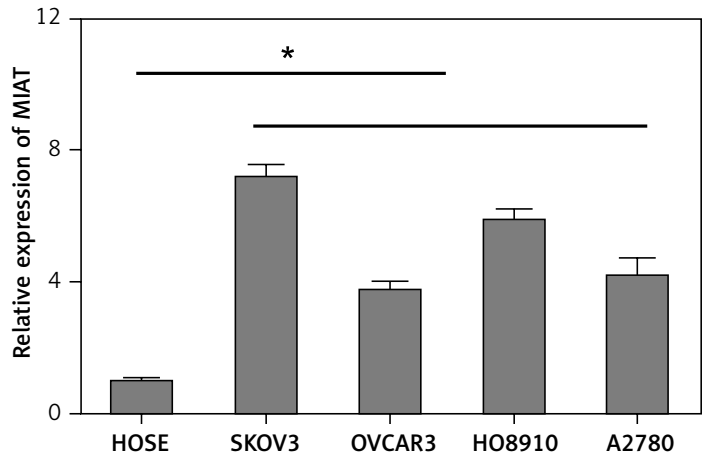

Figure 1. LncRNA MIAT expression is upregulated in EOC tissues and cell lines. A - QRT-PCR analysis of MIAT expression in EOC tissues. B - Relative expression of MIAT was determined by qRT-PCR in EOC cell lines (SKOV3, OVCAR3, HO8910, and A2780) and human ovarian surface epithelial (HOSE) cells. C - The Kaplan-Meier curves showed that EOC patients with high MIAT expression had poor overall survival ${ }^{*} P<0.05$. 

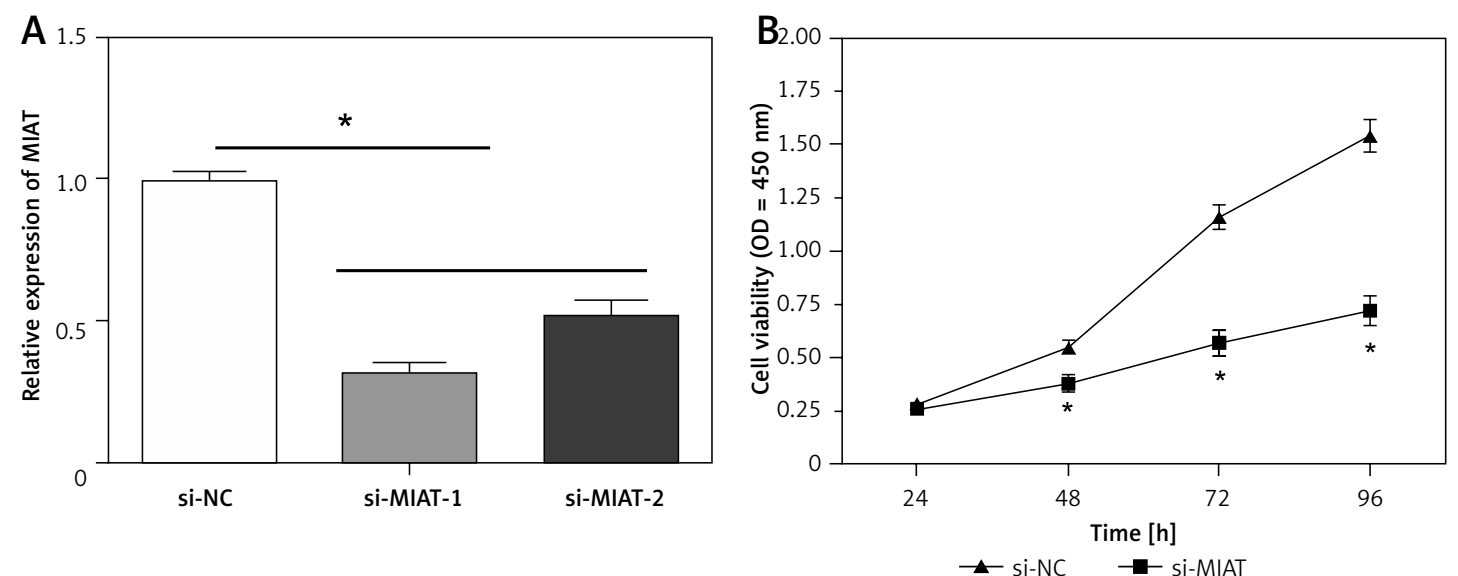

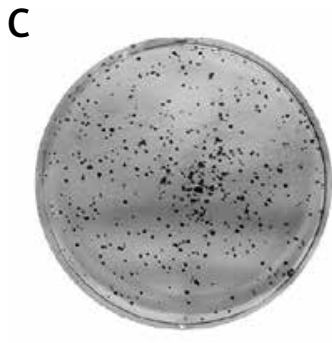

si-NC

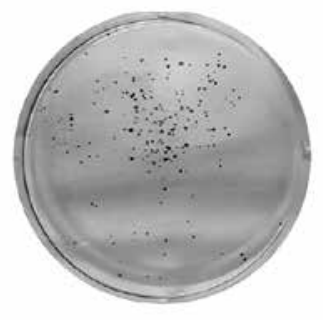

si-MIAT
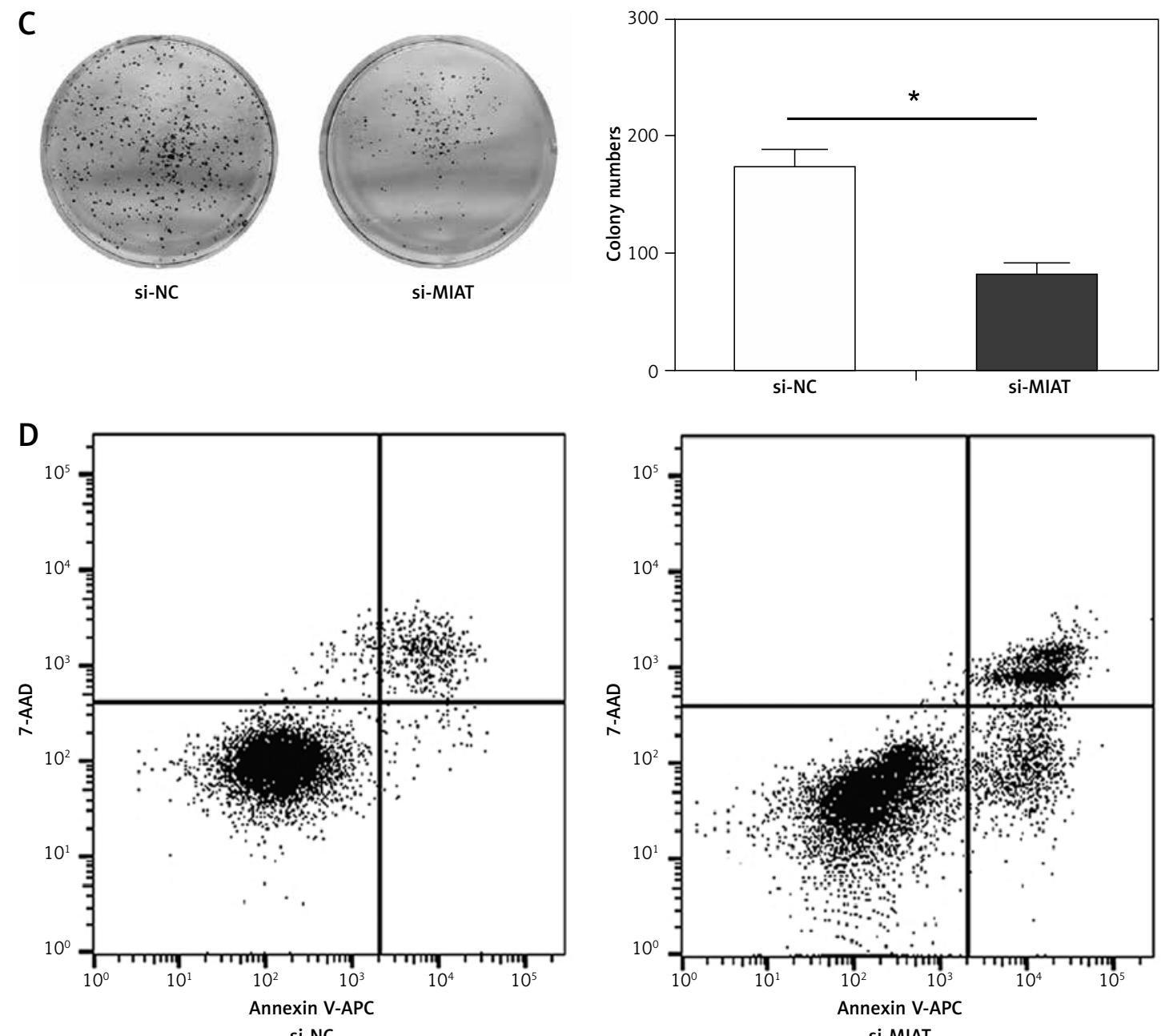

si-NC

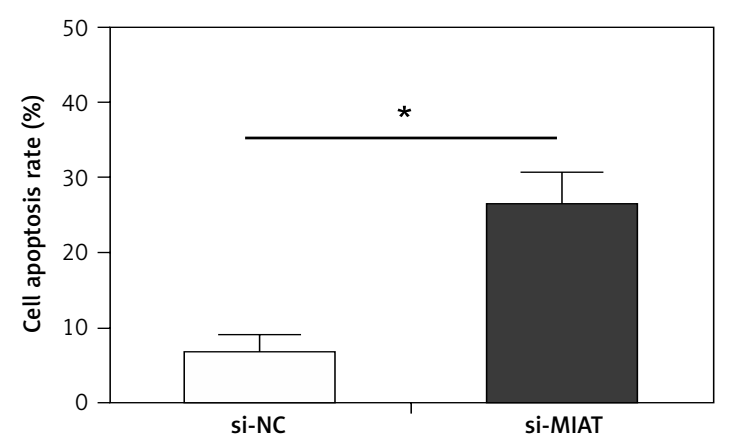

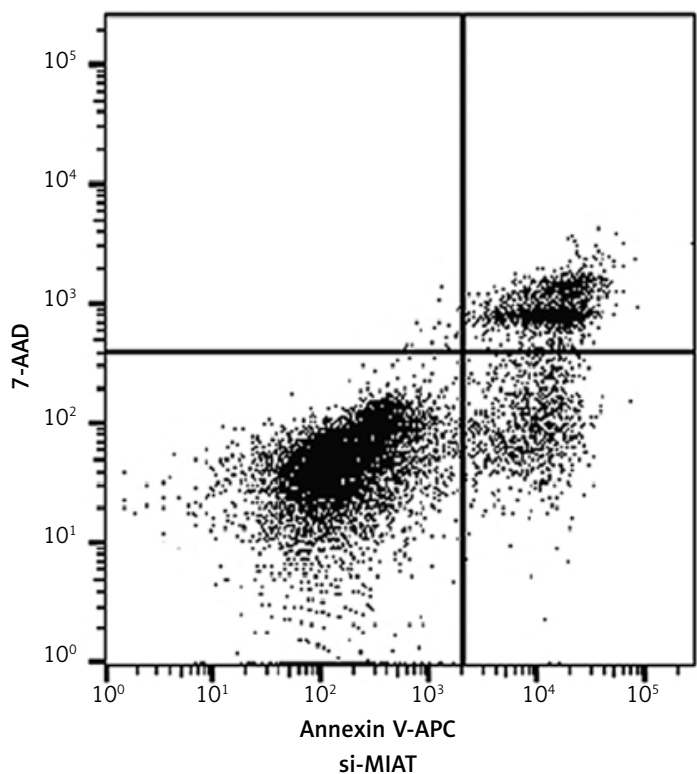

Figure 2. LncRNA MIAT knockdown suppressed EOC cell proliferation and induced cell apoptosis. A - LncRNA MIAT knocked down in SKOV3 cells transfected with si-MIAT. B, C - Cell proliferation ability and colony formation ability were detected by CCK- 8 assays and colony formation assays in SKOV3 cells transfected with si-MIAT or si-NC. D - Relative cell apoptosis was evaluated by flow cytometry assays in SKOV3 cells transfected with Si-MIAT or si-NC

${ }^{*} p<0.05$. 
fied EOC patients into a high MIAT group $(n=27)$ and a low MIAT group $(n=26)$. Kaplan-Meier survival analysis showed that patients with higher MIAT expression had significantly reduced overall survival compared with patients with lower MIAT expression (Figure $1 \mathrm{C} ; p<0.05)$. Those data indicated that MIAT plays important roles in EOC progression.

\section{LncRNA MIAT knockdown inhibited EOC cell proliferation}

To clarify the role of MIAT in EOC cell proliferation and apoptosis, si-MIAT was transfected into SKOV3 cells, and the transfection efficiency was determined by qRT-PCR (Figure $2 \mathrm{~A} ; p<0.05$ ). CCK-8 assays showed that MIAT inhibition significantly decreased SKOV 3 cell proliferation (Figure 2 B; $p<0.05)$. Colony formation assays showed that the clonogenic survival was inhibited in si-MIAT transfected SKOV3 cells (Figure 2 C; $p<0.05$ ). Furthermore, flow cytometric analysis revealed that MIAT inhibition markedly promoted cell apoptosis in SKOV 3 cells (Figure $2 \mathrm{D} ; p<0.05$ ). Thus, our data indicated that MIAT suppression might inhibit EOC cell proliferation by inducing EOC cell apoptosis.

\section{MIAT directly interacted with miR-330-5p}

Increasing evidence has demonstrated that IncRNA might act as a molecular sponge in regulating the biological functions of miRNA [12]. In the present study, bioinformatics prediction (DIANA) results showed that the MIAT sequence harbors a putative miR-330-5p binding region (Figure $3 \mathrm{~A}$ ).

A

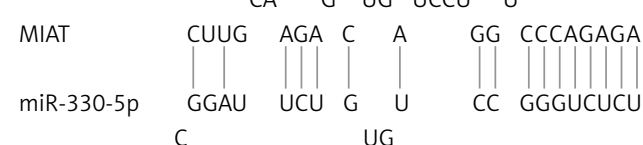

B

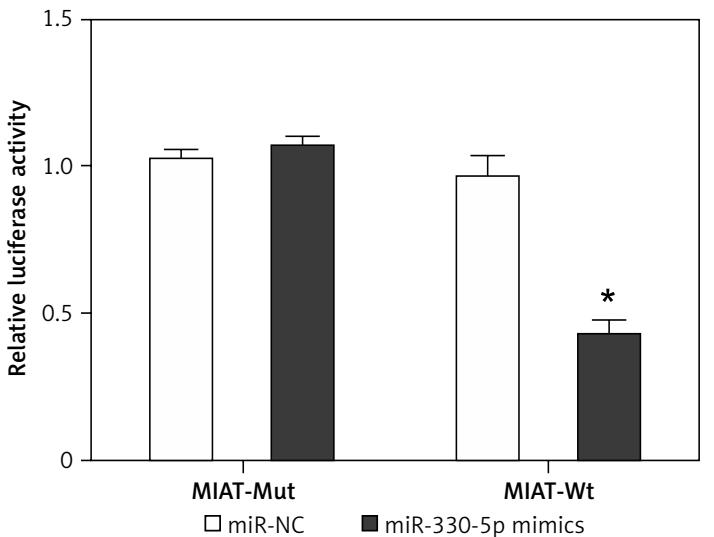

C

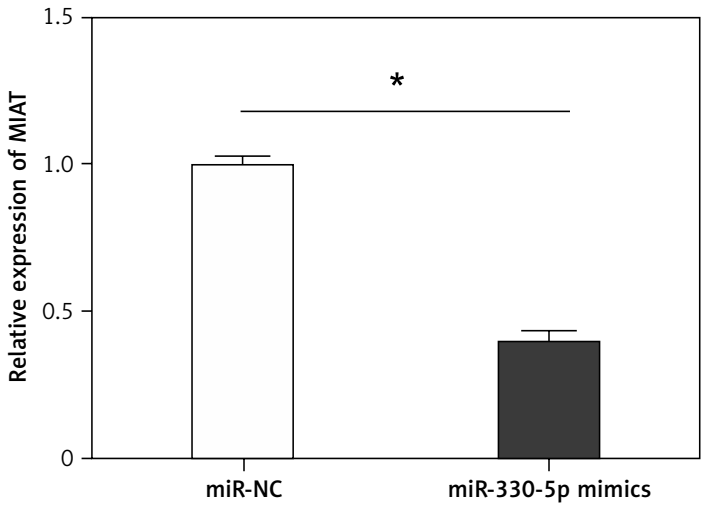

D

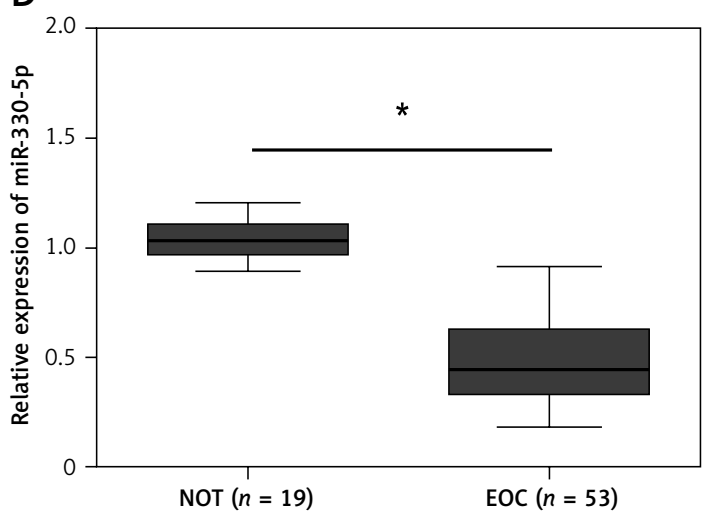

E

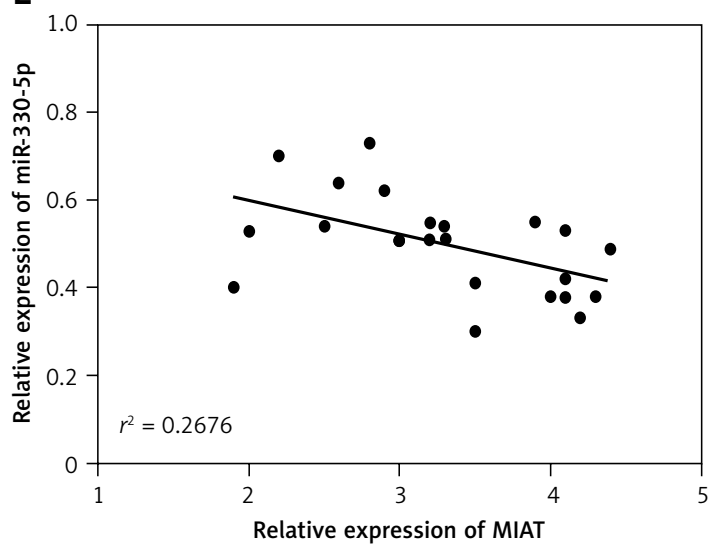

Figure 3. LncRNA MIAT act as a direct target of miR-330-5p. A - The sequence of the predicted miR-330-5p targeting site within MIAT 3'-UTR. B - MiR-330-5p obviously repressed the luciferase activity of the pMIR-MIAT-Wt but not of pMIR-MIAT-Mut in HEK293T cells. C - QRT-PCR analysis of MIAT expression in EOC cells transfected with miR-330-5p mimics. D - QRT-PCR analysis determined miR-330-5p expression in EOC tissues. $\mathbf{E}-$ The correlation analysis showed that miR-330-5p expression was negatively associated with MIAT expression in EOC tissues ${ }^{*} P<0.05$. 


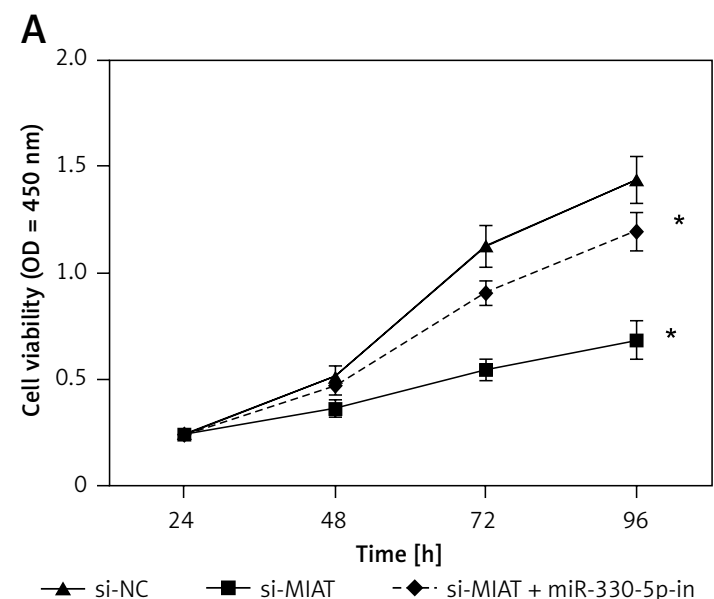

B

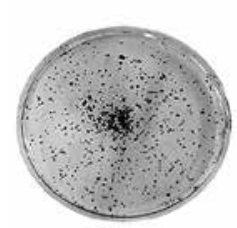

si-NC

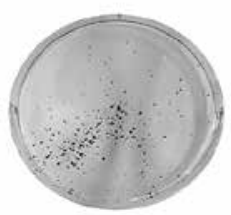

si-MIAT

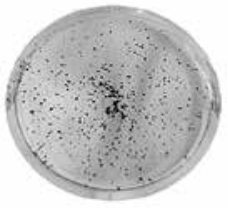

Si-MIAT + miR-330-5p-in

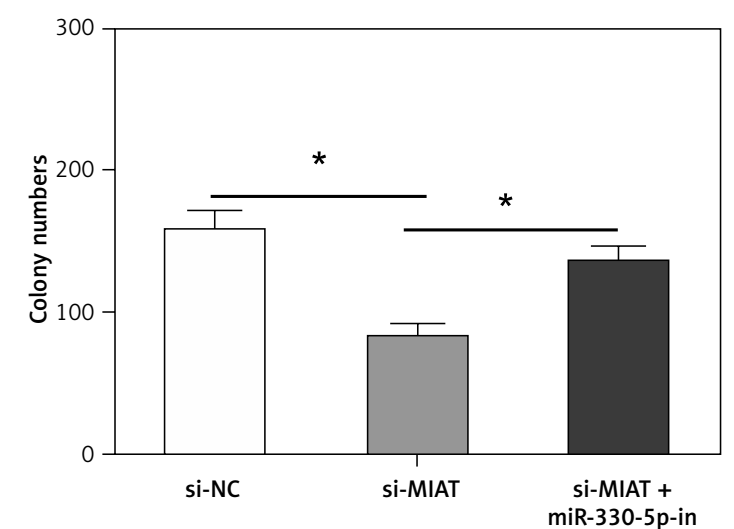

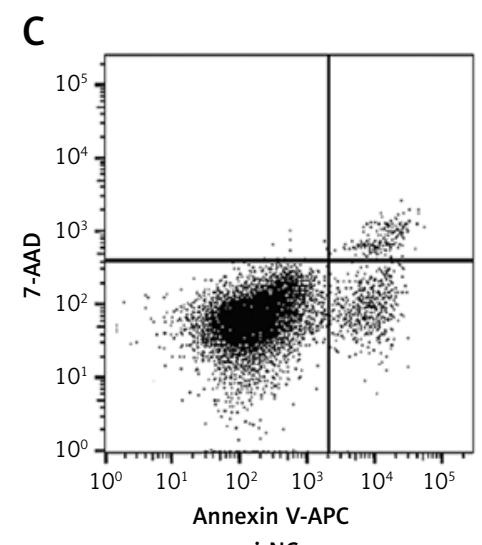

si-NC

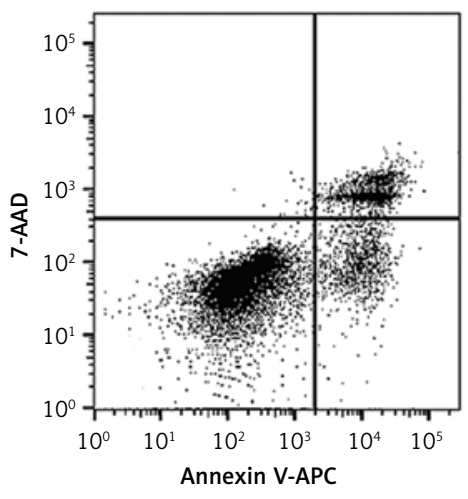

si-MIAT

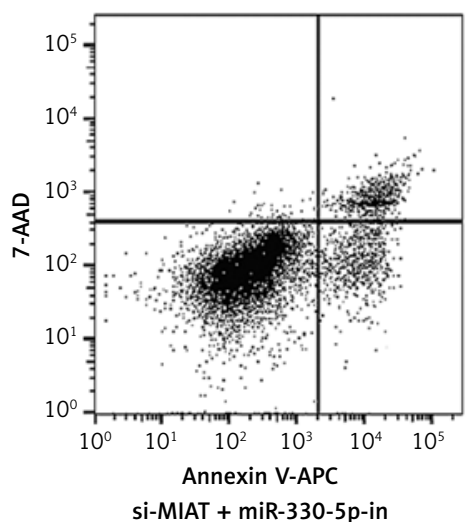

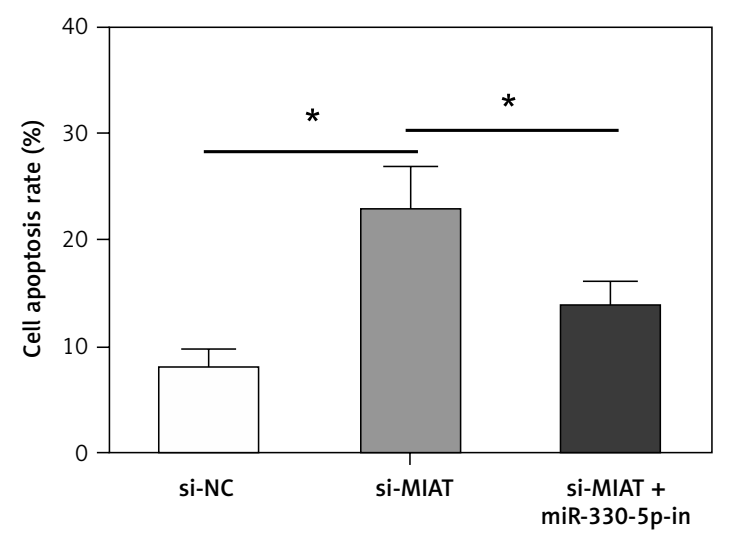

Figure 4. MiR-330-5p inhibitor reversed cell proliferation and apoptosis ability induced by si-MIAT. A, B - Cell proliferation and colony formation ability were determined in SKOV3 cells transfected with si-MIAT and miR-330-5p inhibitors + si-MIAT. C - Cell apoptosis was determined in SKOV3 cells transfected with si-MIAT and miR-330-5p inhibitors + si-MIAT ${ }^{*} P<0.05$. 
To identify whether MIAT is target of miR-330-5p, the wild type sequence of MIAT (Wt-MIAT) or the mutant (Mut-MIAT) with the miR-330-5p recognition sequence was subcloned into the pMIR luciferase reporter, and then transfected into HEK293T cells together with miR-330-5p mimics and miR-NC. The results showed that miR-330-5p mimics decreased the luciferase activity of Wt-MIAT, but not the luciferase activity of Mut-MIAT (Figure $3 \mathrm{~B}$; $p<0.05)$. Furthermore, we explored the correlation between MIAT expression and miR-330-5p. The results showed that MIAT expression was downregulated by transfection with miR-330$5 p$ mimics (Figure $3 c ; p<0.05$ ). In addition, the results showed that miR-330-5p was downregulated in EOC tissues and was inversely correlated with the expression of MIAT (Figures $3 \mathrm{D}, \mathrm{E}$; $p<0.05)$. These results indicated that miR-330-5p could directly bind to MIAT and negatively regulate MIAT expression in EOC.

\section{MIAT affected EOC cell proliferation and apoptosis by regulating miR-330-5p}

In order to explore whether the effect of MIAT on EOC tumorigenicity is mediated by miR-330$5 p$, si-MIAT and miR-330-5p inhibitors + si-MIAT were transfected into SKOV3 cells. CCK-8 assays and colony formation assays showed that miR-330-5p inhibitor abrogated the effect of si-MIAT in reducing cell viability (Figures $4 \mathrm{~A}, \mathrm{~B}$; $p<0.05)$. Meanwhile, the alterations in EOC cell apoptosis caused by silencing MIAT were also rescued by miR-330-5p inhibitors (Figure $4 \mathrm{C}$; $p<0.05)$. Therefore, our study indicated that MIAT could act as an endogenous sponge by binding to miR-330-5p in EOC cells.

\section{Discussion}

Despite the great therapeutic advances made in EOC, including surgical resection and adjuvant therapy, the long-term prognosis of patients with distant metastases remains unfavorable. Increasing numbers of studies have suggested that IncRNAs could serve as effective therapeutic targets for cancer treatment [13]. For example, Feng et al. found that RMRP might act as an oncogene and could be used as a therapeutic target for the treatment of glioma [14]. Zhang et al. showed that upregulation of IncRNA MALAT1 correlated with tumor progression and poor prognosis in clear cell renal cell carcinoma [15]. Recently, studies showed that IncRNA MIAT has an oncogenic effect in many cancers. For example, Lai et al. indicated that MIAT promoted non-small cell lung cancer proliferation and metastasis through MMP9 activation [16]. Luan et al. showed that MIAT promoted breast cancer progression and functions as a ceRNA to regulate DUSP7 expression by sponging miR-155-5p [17]. Li et al. showed that IncRNA MIAT regulated cell biological behaviors in gastric cancer through a mechanism involving the miR-29a-3p/HDAC4 axis [18]. However, the role of MIAT in EOC is still unclear.

In the present study, we found that MIAT expression was highly expressed in EOC tissues and cell lines. Kaplan-Meier survival analysis showed that patients with high MIAT expression had significantly reduced overall survival compared with patients with low MIAT expression. Moreover, we explored the role of MIAT in EOC cells. Our data showed that MIAT knockdown inhibited EOC cell proliferation and colony formation and induced cell apoptosis. Thus, those results indicated that MIAT was involved in EOC progression.

MiR-330-5p was down-regulated in various tumors, and regulated the development of tumor cells [19]. For example, Su et al. showed that miR330-5p suppressed cell proliferation and invasion in cutaneous malignant melanoma [20]. Kong et al. found that miR-330-5p overexpression repressed cell growth of non-small cell lung cancer by inhibiting NOB1 expression [21]. Bibby et al. indicated that miR-330-5p acted as a putative modulator of neoadjuvant chemoradiotherapy sensitivity in oesophageal adenocarcinoma [22]. In the present study, our data showed that miR-330-5p was downregulated in EOC tissues, and downregulated expression of miR-330-5p was negatively corrected with MIAT expression in EOC tissues.

Recently, studies have reported that there is a novel regulatory mechanism between IncRNAs and miRNAs. IncRNA might function as a ceRNA or a molecular sponge in modulating miRNA [23]. For example, Cui et al. showed that upregulated IncRNA SNHG1 contributed to progression of non-small cell lung cancer through inhibition of miR-101-3p and activation of the Wnt/ $\beta$-catenin signaling pathway [24]. Wang et al. indicated that IncRNA XIST exerted oncogenic functions in human glioma by targeting miR-137 [25]. Xie et al. suggested that IncRNA ZFAS1 sponged miR-484 to promote cell proliferation and invasion in colorectal cancer [26]. So we hypothesized that MIAT also has similar effects on EOC progression. Bioinformatics analysis found that the MIAT sequence harbors a putative miR-330-5p binding region. Luciferase activity assays confirmed the direct binding of MIAT to miR-330-5p. QRT-PCR showed that miR-330-5p mimics significantly decreased MIAT expression in EOC cells. Furthermore, we explored whether the effect of MIAT on EOC tumorigenicity is mediated by miR-330-5p. CCK-8 assays and colony formation assays showed that miR-330-5p inhibitor abrogated the effect of Si-MIAT in reducing EOC cell proliferation ability. Meanwhile, the alterations in EOC cell apoptosis caused by silencing MIAT were also rescued by miR-330-5p inhibitors. 
In conclusion, in this study, we demonstrated that IncRNA MIAT acted as an oncogene in EOC progression by suppressing the expression of miR330-5p. These data suggested that the MIAT/miR330-5p axis might act as a potential therapeutic target for the treatment of EOC patients.

\section{Conflict of interest}

The authors declare no conflict of interest.

\section{References}

1. Jemal A, Bray F, Center MM, et al. Global cancer statistics. CA Cancer J Clin 2011; 61: 69-90.

2. Holschneider $\mathrm{CH}$, Berek JS. Ovarian cancer: epidemiology, biology, and prognostic factors. Semin Surg Oncol 2000; 19: 3-10.

3. Morgan RJ, Alvarez RD, Armstrong DK, et al. Epithelial ovarian cancer. J Natl Compr Canc Netw 2011; 9: 82-113.

4. Mercer TR, Dinger ME, Mattick JS. Long non-coding RNAs: insights into functions. Nat Rev Genet 2009; 10: 155-9.

5. Zhu FF, Zheng FY, Wang HO, et al. Downregulation of IncRNA TUBA4B is associated with poor prognosis for epithelial ovarian cancer. Pathol Oncol Res 2018; 24: 419-25.

6. Jin Y, Feng SJ, Qiu S, et al. LncRNA MALAT1 promotes proliferation and metastasis in epithelial ovarian cancer via the PI3K-AKT pathway. Eur Rev Med Pharmacol Sci 2017; 21: 3176-84.

7. Zhang Y, Dun Y, Zhou S, et al. LncRNA HOXD-AS1 promotes epithelial ovarian cancer cells proliferation and invasion by targeting miR-133a-3p and activating Wnt/ beta-catenin signaling pathway. Biomed Pharmacother 2017; 96: 1216-21.

8. Ishii N, Ozaki K, Sato H, et al. Identification of a novel non-coding RNA, MIAT, that confers risk of myocardial infarction. J Hum Genet 2006; 51: 1087-99.

9. Yan B, Liu J, Yao J, et al. IncRNA-MIAT regulates microvascular dysfunction by functioning as a competing endogenous RNA. Circ Res 2015; 116: 1143-56.

10. Liao J, He Q, Li M, et al. LncRNA MIAT: myocardial infarction associated and more. Gene 2016; 578: 158-61.

11. Stitt AW, Curtis TM, Chen M, et al. The progress in understanding and treatment of diabetic retinopathy. Prog Retin Eye Res 2016; 51: 156-86.

12. Varga M, Sixta B, Bem R, et al. Application of gentamicin-collagen sponge shortened wound healing time af ter minor amputations in diabetic patients - a prospective, randomised trial. Arch Med Sci 2014; 10: 283-7.

13. Prensner JR, Chinnaiyan AM. The emergence of IncRNAs in cancer biology. Cancer Discov 2011; 1: 391-407.

14. Feng $\mathrm{W}$, Li $L, X U X$, et al. Up-regulation of the long non-coding RNA RMRP contributes to glioma progression and promotes glioma cell proliferation and invasion. Arch Med Sci 2017; 13: 1315-21.

15. Zhang H, Yang F, Chen SJ, et al. Upregulation of long non-coding RNA MALAT1 correlates with tumor progression and poor prognosis in clear cell renal cell carcinoma. Tumor Biol 2015; 36: 2947-55.

16. Lai IL, Yang CA, Lin PC, et al. Long noncoding RNA MIAT promotes non-small cell lung cancer proliferation and metastasis through MMP9 activation. Oncotarget 2017; 8: 98148-62.
17. Luan T, Zhang X, Wang S, et al. Long non-coding RNA MIAT promotes breast cancer progression and functions as ceRNA to regulate DUSP7 expression by sponging miR-155-5p. Oncotarget 2017; 8: 76153-64.

18. Li Y, Wang K, Wei Y, et al. IncRNA-MIAT regulates cell biological behaviors in gastric cancer through a mechanism involving the miR-29a-3p/HDAC4 axis. Oncol Rep 2017; 38: 3465-72.

19. Tréhoux S, Lahdaoui F, Delpu Y, et al. Micro-RNAs miR29a and miR-330-5p function as tumor suppressors by targeting the MUC1 mucin in pancreatic cancer cells. Biochim Biophys Acta 2015; 1853: 2392-403.

20. Su B, Zhou S, Gan C, et al. MiR-330-5p regulates tyrosinase and PDIA3 expression and suppresses cell proliferation and invasion in cutaneous malignant melanoma. J Surg Res 2016; 203: 434-40.

21. Kong R, Liu W, Guo Y, et al. Inhibition of NOB1 by microRNA-330-5p overexpression represses cell growth of non-small cell lung cancer. Oncol Rep 2017; 38: 2572-80.

22. Bibby BAS, Reynolds JV, Maher SG. MicroRNA-330-5p as a putative modulator of neoadjuvant chemoradiotherapy sensitivity in oesophageal adenocarcinoma. PloS One 2015; 10: e0134180.

23. Li JH, Liu S, Zhou H, et al. starBase v2. 0: decoding miRNA-ceRNA, miRNA-ncRNA and protein-RNA interaction networks from large-scale CLIP-Seq data. Nucleic Acids Res 2013; 42: D92-7.

24. Cui Y, Zhang F, Zhu C, et al. Upregulated IncRNA SNHG1 contributes to progression of non-small cell lung cancer through inhibition of miR-101-3p and activation of Wnt/beta-catenin signaling pathway. Oncotarget 2017; 8: 17785-94.

25. Wang Z, Yuan J, Li L, et al. Long non-coding RNA XIST exerts oncogenic functions in human glioma by targeting miR-137. Am J Transl Res 2017; 9: 1845-55.

26. Xie S, Quanxing G, Wang X, Sun X, Kang Y. Long non-coding RNA ZFAS1 sponges miR-484 to promote cell proliferation and invasion in colorectal cancer. Cell Cycle 2018; 17: 154-61 